\title{
Calidad de vida en docentes de educación pública superior*
}

\author{
María de los Angeles Romero Espinoza ${ }^{1}$ \\ (i) orcid.org/0000-0003-2782-0212 \\ Universidad Estatal de Sonora, México \\ Jesús Francisco Laborín Alvarez ${ }^{2}$ \\ (1D) orcid.org/0000-0003-0747-6426 \\ Centro de Investigación en Alimentación y Desarrollo A.C., México
}

DOI: http://dx.doi.org/10.17081/eduhum.18.31.1375

Recibido: 5 de marzo de 2016

Aceptado: 1 de agosto de 2016

\section{Life quality of high public education teachers}

Palabras clave:
Calidad de vida,
Educación superior,
Condiciones objetivas de vida,
Bienestar subjetivo,
Enfrentamiento a los problemas.

\section{Keywords:}

Life quality, Higher Education, Object life conditions,

Subject welfare, Problem facing.

\begin{abstract}
Resumen
A partir de una revisión bibliográfica en torno a la calidad de vida (CV), la evolución del concepto y su medición, se propone un modelo alternativo que incorpora sus componentes objetivo y subjetivo y además, considera al enfrentamiento a los problemas como una variable que también contribuye a explicar la CV de las personas. En este caso particular, sobre docentes de Educación pública superior, pocos estudios se han encontrado sobre sus condiciones objetivas de vida y de los recursos psicológicos con que cuentan para enfrentar las situaciones estresantes derivadas de su trabajo académico y de otros ámbitos de sus vidas.
\end{abstract}

\begin{abstract}
After reviewing the bibliography in relation to life quality (LQ), the evolving and measurement of the concept, an alternative model is proposed that includes an object and a subject component, and additionally considers, in itself, the facing of problems as a variable that contributes to explain the LQ (Life Quality) of people. In this particular case, concerning High Public Education Teachers, only a few research studies have been found about their objective life conditions and psychological resources on which teachers must rely on for facing stress situations derived from their Academic jobs and from any other life event.
\end{abstract}

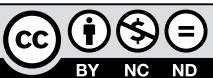

Referencia de este artículo (APA): Romero, M. A. \& Laborín, J. F. (2016). Calidad de vida en docentes de educación pública superior. Revista Educación y Humanismo, 18(31), 205-224. http://dx.doi.org/10.17081/eduhum.18.31.1375

\footnotetext{
* Artículo resultado de investigación asociado al proyecto en desarrollo "Calidad de vida en docentes de educación pública superior", desde enero de 2014 y que concluirá en diciembre de 2017, que cuenta con el respaldo institucional del Centro de Investigación en Alimentación y Desarrollo A.C. y de la Universidad Estatal de Sonora (México).

1. Doctorante en Desarrollo Regional, Magíster en Desarrollo Humano en Organizaciones y Ciencias Sociales, Licenciada en Ciencias de la Comunicación. Profesora de la Universidad Estatal de Sonora, México. Email: marielos.romero@ues.mx

2. Doctor en Ciencias Sociales, Magíster en Psicología Social, Psicólogo. Profesor titular del Centro de Investigación en Alimentación y Desarrollo A.C., México. Email: laborin@ciad.mx
} 
A partir de la década de los noventa, algunos organismos internacionales asumieron el papel de orientadores e impulsores de políticas para transformar la educación superior (Villaseñor, 2003).

Según Gil (2012), se pueden ubicar entre 1990 y 2010 tres impactos que han alterado el panorama de la educación superior en México: una reducción importante de los salarios en las instituciones por las crisis económicas; reformas constitucionales que dejaron en manos de las propias universidades establecer los criterios de ingreso, promoción y permanencia de sus profesores; y otorgar, de manera focalizada, ingresos adicionales mediante la evaluación individual del desempeño de los académicos.

\section{Planteamiento del problema}

Junto con todos los cambios derivados de las crisis económicas, las restricciones en el financiamiento de las universidades y los cambios en los modelos educativos, se diversifican las funciones y responsabilidades para el docente de educación superior.

Al profesor universitario se le comienza a exigir mayor preparación y compromiso con el diseño de su programa de formación, así como en el de sus multivariadas estrategias metodológicas, donde el uso y el manejo de las tecnologías de información y comunicación (TIC) son indiscutibles (Ruiz, Mas, Tejada \& Navío, 2008).

Este panorama está vinculado con los ingre- sos de los docentes de educación superior. Al respecto, González, Brunner y Salmi (2013), basándose en otros autores, afirman que en muchos países desarrollados los salarios de académicos están cayendo por debajo de los salarios de otras profesiones que requieren un nivel educacional comparable. Los autores plantean que países como Japón, Alemania, Israel y Estados Unidos tendrán dificultades para atraer a los jóvenes más talentosos en el futuro, a menos de que los salarios en los rangos bajos de la jerarquía mejoren y que el personal más joven sea disuadido por la creciente presión derivada de una mayor carga docente y del creciente énfasis en el número de publicaciones.

En el caso de México, mientras los salarios tabulares han permanecido por años prácticamente estancados, el desempeño individual va en ascenso. Prueba de ello es que hoy se cuenta con más miembros en el Sistema Nacional de Investigadores (SNI) y con más profesores de tiempo completo (PTC) reconocidos por el Programa de Mejoramiento del Profesorado (Promep) que hace media década; también el número de académicos que se beneficia del programa de estímulos va en aumento. Entretanto, las condiciones de trabajo objetivas y subjetivas del docente investigador no tienden a una mejora general. Aun cuando la institución se ha hecho cargo de proporcionar espacios de trabajo más adecuados y funcionales gestionando recursos, el salario se fragmenta en una proporción fija y otra variable ligada a la productividad, y esto implica mantener un ritmo de trabajo intenso de continuo, a 
riesgo de una reducción de las remuneraciones (Acosta \& Aguilar, 2013).

Es necesario tener en cuenta que las estructuras organizacionales de las universidades están consideradas entre las más complejas, ambiguas y anárquicas, lo que se contrapone a las visiones tradicionales de organización (Barba, 2012; De Vries \& Ibarra, 2004). En ese sentido, la transformación de la universidad se encuentra en la reinvención de su identidad en el imaginario social y con ello, la de sus prácticas y modos de actuar. El debate está en si estas transformaciones suponen el desplazamiento de la universidad como referente cultural básico de la sociedad, hacia la adquisición del estatuto de las empresas que prestan algún servicio a la sociedad (Ibarra, 2003, 2005).

Para Comas y Rivera (2011) esta transformación ha traído consigo una serie de consecuencias, específicamente sobre el sistema de estímulos, que generaron efectos positivos y negativos o inesperados:

a) Efectos positivos: una política salarial diferente para los académicos a través de recompensas otorgadas previa evaluación; el doble de programas evaluados y acreditados de calidad; fomento a la permanencia, la productividad y la dedicación de tiempo completo; impulso a la carrera académica como una trayectoria profesional; y mejoramiento en la habilitación de los profesores.

b) Efectos negativos o inesperados: los estímulos económicos no tuvieron influencia sobre la etapa jubilatoria en las universidades; la falta de actualización de los procesos para evaluar a los académicos; los salarios del personal académico continuaron rezagados; el favorecimiento a la acumulación de los trabajos por encima de su calidad; la priorización de actividades que podrían ser contabilizadas para la obtención del estímulo; la competitividad generada no tuvo repercusiones significativas en la eficiencia terminal ni en la permanencia de alumnos en las instituciones. El interés económico se sobrepuso a la esencia del trabajo académico, afectando principalmente la docencia.

Sin duda la transformación que ha sufrido la universidad ha traído consigo consecuencias en el cambio de sus modelos educativos, en las funciones de los docentes, así como en el perfil de competencias que de estos se demanda; pero además, ha impactado sus dinámicas de trabajo académico y su calidad de vida laboral. Algunos estudios dan cuenta de ello:

Terán y Botero (2011) en su análisis del capitalismo organizacional con relación a la calidad de vida laboral en la docencia universitaria, concluyeron que los efectos más relevantes fueron: el aumento del compromiso económico, la imagen del profesional de la universidad desvirtuada en el mercado y el rol docente subordinado al rol investigador. $\mathrm{Y}$ sugieren que es prioritaria una revisión de las condiciones contractuales, especialmente de la estabilidad laboral, así como la consideración de la llamada carga laboral, pues- 
to que se está generando un desequilibrio entre la vida personal y la laboral, y, por ende, una disminución de la calidad de vida.

La Universidad Autónoma Metropolitana encontró en un estudio (Martínez, Méndez \& Murata, 2011) que cubrir las exigencias para alcanzar becas o pertenecer al SNI asocian daños a la salud de los docentes. De estas, las más importantes fueron: la sobrecarga de trabajo, la prolongación de la jornada y las posiciones incómodas; cinco de siete consecuencias de la participación en el programa se perfilaron como negativas y se asociaron estadísticamente con trastornos tales como el estrés, trastornos músculo-esqueléticos, la fatiga, la disfonía, la ansiedad y los trastornos del sueño.

Otro estudio realizado recientemente de la Universidad Autónoma de Nuevo León analizó las diferencias en el nivel de estímulos y las variables de género, nivel del SNI, pertenencia a un cuerpo académico (CA), área de conocimiento y nivel de titularidad. En el caso del sueldo, las únicas relaciones en las que mostraron diferencias estadísticamente significativas fueron aquellas entre el nivel de titularidad y el nivel del SNI. Los resultados del estudio reflejaron que pertenecer a un CA, el género o el área de conocimiento no son variables que expliquen diferencias en el nivel de estímulo de los investigadores (Ramos, Sieglin \& Zúñiga, 2013).

Por su parte, Urquidi y Rodríguez (2010) desarrollaron una investigación con docentes de educación superior ubicados en el noroeste de México, la cual se llevó a cabo con el propósito de explorar el grado de estrés que este grupo experimenta como producto del ejercicio de su profesión. Los resultados muestran que las 10 situaciones más estresantes se relacionan con los programas de estímulo académico que operan en las instituciones mexicanas desde 1990. Las dimensiones que incorporan situaciones estresantes son: a) la sobrecarga de trabajo y las limitaciones de tiempo para cumplir con las tareas académicas, b) el ambiente institucional, c) el reconocimiento que se otorga a través de los programas de estímulos y d) las condiciones físicas. De estos cuatro factores, la media más alta se identifica en la dimensión vinculada con el reconocimiento.

A pesar de las casi dos décadas de operación de los programas de estímulos, los académicos y las académicas mexicanas no han podido adaptarse a sus exigencias, lo que inevitablemente lleva a experimentar estrés en diferentes grados Urquidi y Rodríguez (2010). En contraste, Terán y Botero (2011) concluyen que al explorar sobre las estrategias y prácticas de afrontamiento individual y colectivo de las demandas organizacionales, más del $60 \%$ de la población docente encuestada se adapta en gran medida a las demandas de la organización. Es decir, se presenta una alta tendencia a aceptar las normas, los cambios y los ritmos de trabajo.

En otro estudio se analizó cómo las políticas neoliberales instrumentadas en las universidades 
han configurado condiciones laborales precarias, donde la interacción humana se torna conflictiva y difícil y sienta las bases para el desarrollo del burnout, el cual es entendido como una respuesta frente al estrés laboral crónico, que incluye agotamiento, despersonalización y falta de realización personal, y que se ha constituido en uno de los trastornos que mayor impacto están teniendo sobre la salud de los trabajadores académicos (Martínez \& Preciado, 2009).

En suma, teniendo como consigna el mejoramiento de la calidad, las políticas gubernamentales fueron condicionando la evaluación permanente al financiamiento para las IES y a las remuneraciones adicionales para los docentes (Ibarra \& Porter, 2007a/2007b), con el consiguiente aumento de exigencias que de alguna manera impactan la esencia de su identidad como académicos, incluso su salud en algunos casos. De ahí que se considere necesario profundizar acerca de cómo ellos relacionan las condiciones objetivas de vida, los niveles de satisfacción de los docentes de tiempo completo de educación pública de nivel superior y la manera o estilo como asumen los problemas en los diferentes dominios de sus vidas.

Lo anterior implica centrarse principalmente en la relación de tres constructos: calidad de vida (Veenhoven, 1994), bienestar subjetivo (Diener, Suh, Lucas \& Smith, 1999; Diener, 2000) y enfrentamiento a los problemas (Lazarus \& Folkman, 1984, citados en Folkman, Lazarus, Dunkel-Schetter, DeLongis, \& Gruen, 1986).
El desarrollo del país exige ponerse metas de mejoramiento en todos los ámbitos de la vida social, particularmente en el ámbito laboral, donde se requiere elevar los estándares de calidad del empleo y de las condiciones de trabajo para que la competitividad internacional esté basada en un uso inteligente de los recursos disponibles. En este marco, los recursos humanos juegan un papel fundamental (Gobierno de Chile, 2002). Entretanto, en los últimos años México ha enfrentado retos de competitividad, pero registra las más bajas calificaciones en el rubro de innovación. Por otro lado, aun cuando los procesos de evaluación a instituciones de educación superior (IES) han crecido consistentemente contribuyendo a la mejora de la calidad educativa y a los indicadores educativos nacionales, esto no ha representado una mejora significativa en las actividades de innovación y responsabilidad de las IES (Malacara, Cázares, Galván \& Ramírez, 2014).

La línea más fructífera de investigación en torno a los cambios en la universidad debería ser el estudio de las consecuencias sociales y simbólicas de la reorganización institucional, así como de sus consecuencias en la reinvención de los modos de existencia de los docentes, protagonistas centrales del devenir universitario. Si el docente es el agente clave en el desarrollo cotidiano de la vida de la universidad, este debería ser uno de los primeros convocados para realizar dicha transformación, toda vez que es uno de los principales afectados (Ibarra, 2002; López \& Restrepo, 2011), y en razón de su condición de 
agente de cambio fundamental tanto al interior de la universidad como al exterior, pues es quien forma a los futuros profesionistas que habrán de impulsar o no el desarrollo de su entorno.

Por consiguiente, profundizar sobre la calidad de vida y contar con un modelo explicativo de las relaciones entre las variables derivadas de las condiciones objetivas de vida, el bienestar subjetivo y el enfrentamiento a los problemas, podría contribuir a la gestión más eficiente de estos recursos humanos clave en la universidad, $\mathrm{y}$, por ende, contar con los académicos para recuperar la verdadera esencia de las universidades, su función social, la discusión, reflexión crítica y las propuestas innovadoras en beneficio del desarrollo del país.

\section{Revisión y situación actual del concepto de calidad de vida \\ Conforme al PNUD (1994, citado en Pérez-} Luco, Alarcón \& Zambrano, 2004), el propósito del desarrollo consiste en crear una atmósfera en que todos puedan aumentar su capacidad y las oportunidades puedan ampliarse para las generaciones presentes y futuras. Para Delgado y Salcedo (2008), esto debería implicar la creación de un ambiente favorable en el que las personas tengan la posibilidad de alcanzar una vida prolongada, saludable y creativa, que trae consigo la longevidad, los logros en educación y los recursos materiales.

De acuerdo con el Informe de Desarrollo Humano del 2013 (Programa de las Naciones Uni- das para el Desarrollo [PNUD], 2014), las clasificaciones del IDH fueron de: muy alto, alto, medio (47 países en cada clasificación) y bajo (46 países). México ocupó la posición número 61 en el grupo de alto IDH, por debajo de Uruguay, Cuba y Panamá en el contexto latinoamericano.

La utilización del concepto de calidad de vida (CV) puede remontarse a Estados Unidos después de la Segunda Guerra Mundial, pero su uso extendido se da a partir de los sesenta, cuando los científicos sociales inician investigaciones en $\mathrm{CV}$ recolectando información y datos objetivos como el estado socioeconómico, nivel educacional o tipo de vivienda; indicadores que muchas veces resultan ser insuficientes, dado que solo eran capaces de explicar un $15 \%$ de la varianza en la CV individual (Urzúa \& Caqueo-Urízar, 2012). En la década siguiente, la investigación se reorientó hacia los indicadores psicológicos o subjetivos (felicidad y satisfacción), que parecían ser la medida más exacta de la CV (Haas, 1999).

Para autores como Fernández-López, Fernández-Fidalgo y Cieza (2010), la primera utilización del concepto fue con la investigación científica de la asistencia social benéfica con los trabajos del grupo de Wolfgang Zapf, a finales de los sesenta. Entonces el concepto fue definido como la correlación existente entre el nivel de vida objetivo de un grupo de población determinado y su correspondiente valoración subjetiva. En las décadas siguientes el término CV se usó indistintamente para nombrar numerosos aspec- 
tos diferentes de la vida como estado de salud, función física, bienestar físico, adaptación psicosocial, bienestar general, satisfacción con la vida y felicidad.

Según Cummins (2004, citado en Urzúa \& Caqueo-Urízar, 2012), el concepto de CV actualmente incorpora tres ramas de las ciencias: Economía, Medicina y Ciencias Sociales. Cada una de estas disciplinas ha promovido el desarrollo desde un punto de vista diferente respecto a la conceptualización de la CV.

La CV relacionada con la salud se usa para designar los resultados concretos de la evaluación clínica y la toma de decisiones terapéuticas (Fernández-López et al., 2010). La Economía hace referencia en dos sentidos: al bienestar de los individuos en una sociedad, ya que se preocupan por las elecciones que realizan; y a las teorías del desarrollo, que se centran en los procesos de crecimiento de una sociedad y su grado de progreso (Delgado \& Salcedo, 2008). La Psicología, por su parte, ha aportado a la investigación de la CV conceptos como la satisfacción vital y el bienestar subjetivo (Tonon, 2016).

Para poder evaluarle, la calidad de vida debe reconocerse como concepto multidimensional que incluye estilo de vida, vivienda, satisfacción en la escuela y en el empleo, así como situación económica. Es por ello que la calidad de vida se conceptualiza de acuerdo con un sistema de valores, estándares o perspectivas que varían de persona a persona, de grupo a grupo y de lugar a lugar (Velarde-Jurado \& Ávila-Figueroa, 2002).
Varios organismos rechazan el ingreso como indicador adecuado del desarrollo humano a partir de dos argumentos principales: el primero, de tipo teórico, señalan que el bienestar de un país no depende del ingreso en sí mismo, sino del uso que se dé a este. El segundo argumento se basa en la constatación empírica de que un alto nivel de desarrollo humano puede lograrse con un ingreso per cápita moderado, y de que un ingreso per cápita elevado no garantiza el desarrollo humano adecuado; por lo que se concluye que el crecimiento del ingreso no asegura el desarrollo humano (Delgado \& Salcedo, 2008). En concordancia con lo anterior, la recuperación del concepto de calidad de vida surge a partir de la crítica a los enfoques economicistas solo centrados en el bienestar material de las sociedades a nivel agregado y que dejan de lado los componentes subjetivos y sociológicos relacionados con el bienestar integral de las personas en su diversidad (Gobierno de Chile, 2002).

Para Veenhoven (1994), el término calidad de vida denota dos significados: la presencia de condiciones consideradas necesarias para una buena vida y la práctica del vivir bien como tal. El primero se usa a nivel societal y se refiere a las condiciones esenciales como alimentación, vivienda y cuidados médicos suficientes. A nivel individual, el término se usa en sus dos acepciones, en tanto pueden o no concurrir ambas condiciones (una persona puede ser rica, poderosa y popular, pero estar afligida y achacosa; o bien, alguien que es pobre, carece de influencias y está aislado, puede tener un buen desarrollo mental y físico). 
Para la Organización Mundial de la Salud (2008, citada en Banda \& Morales, 2012), la CV se refiere a la percepción que un individuo tiene de su lugar en la existencia, en el contexto de la cultura y del sistema de valores en los que vive, y en relación con sus objetivos, expectativas, normas e inquietudes. En su concepción más amplia, la calidad de vida recibe la influencia de factores como empleo, vivienda, acceso a servicios públicos, comunicaciones, urbanización, criminalidad, contaminación del ambiente y otros que conforman el entorno social y que influyen sobre el desarrollo humano de una comunidad (Velarde-Jurado \& Ávila-Figueroa, 2002).

En la revisión realizada por Urzúa y CaqueoUrízar (2012) los conceptos de calidad de vida y bienestar subjetivo concluyeron que no se encontraron definiciones únicas, por lo que ofrecen una clasificación de definiciones por categorías en grupos:

a) $\mathrm{CV}=$ Condiciones de vida. Bajo esta perspectiva, la $\mathrm{CV}$ es equivalente a la suma de los puntajes de las condiciones de vida objetivamente medibles en una persona, tales como salud física, condiciones de vida, relaciones sociales, actividades funcionales u ocupación.

b) $\mathrm{CV}=$ Satisfacción con la vida. Esta categoría considera la CV como un sinónimo de satisfacción personal. Así, la vida sería la sumatoria de la satisfacción en los diversos dominios que la comprenden. Este tipo de definiciones también tiene sus detractores, ya que otorga el principal foco de interés al bienestar subje- tivo dejando de lado las condiciones externas de vida - como su contexto económico, social, cultural, político o incluso de su propio estado de salud-, en el logro de la satisfacción personal.

c) $\mathrm{CV}=$ Condiciones de vida + satisfacción con la vida. Este enfoque conlleva establecer objetivamente condiciones de vida mediante indicadores biológicos, sociales, materiales, conductuales y psicológicos, los que sumados a los sentimientos subjetivos sobre cada área pueden ser reflejados en el bienestar general. Este grupo de definiciones intentaría conciliar la discusión que plantea tomar la $\mathrm{CV}$ desde un punto de vista netamente objetivo o desde uno subjetivo.

d) $\mathrm{CV}=$ Condiciones de vida + satisfacción con la vida * Valores. La CV es definida como un estado de bienestar general que comprende descriptores objetivos y evaluaciones subjetivas de bienestar físico, material, social y emocional, junto con el desarrollo personal y de actividades, todas estas mediadas por los valores personales. Por tanto, en la medida en que se registran cambios en los valores, en las condiciones de vida o en la percepción, así mismo se pueden provocar otros ajustes en los demás factores según un proceso dinámico. En esta categoría de definiciones podría incluirse la planteada por la OMS.

e) $\mathrm{CV}=$ Condiciones de vida + satisfacción con la vida * Evaluación. El nivel de CV percibido por un individuo estaría fuertemente modulado por los procesos cognitivos vinculados a la evaluación que la persona realiza 
tanto de sus condiciones objetivas de vida como del grado de satisfacción con cada una de ellas.

Sin duda resulta necesario realzar la importancia del aspecto psicológico de la calidad de vida, a lo que se le ha llamado bienestar subjetivo (BS), el cual según Palomar (2005) también se le conoce como bienestar, calidad de vida, bienestar psicológico, felicidad y satisfacción en la vida. Los estudios sobre calidad de vida subjetiva durante las últimas décadas utilizan términos como "satisfacción", "bienestar subjetivo" y "felicidad" de manera intercambiable, aunque las escalas para medir estas variables difieran considerablemente entre sí (Cummins \& Cahill, 2000).

Para Diener, Suh, Lucas y Smith (1999), el BS es una categoría amplia de fenómenos que incluye las respuestas emocionales de las personas, dominios de satisfacción y juicios globales de satisfacción con la vida. Los componentes del BS son: el afectivo, constituido por afectos tanto agradables como desagradables; y el cognitivo, que considera los juicios de satisfacción con la vida en dominios específicos, tales como trabajo, familia, salud, amigos y pareja.

Así pues, dado que la calidad de vida implica considerar condiciones de vida, satisfacción personal, valores y la evaluación de los individuos acerca de cómo se sienten con su vida, los diferentes instrumentos e indicadores generados han permitido hacer comparaciones entre países.
En ese sentido, con base en diferentes campos de investigación, Veenhoven (2006) analiza los significados de las medidas comprensivas de la calidad de vida y brinda cuatro ejemplos de estas:

a) Índice de calidad médica de vida. Una de las medidas más comunes en la investigación de la calidad de vida médica es la "SF-36 Health Survey" (Ware, 1996, citado en Veenhoven, 2006). Se trata de un cuestionario sobre limitaciones físicas en las tareas diarias; limitaciones físicas en el rendimiento del trabajo: dolor corporal, salud general percibida, vitalidad; limitaciones físicas y/o emocionales para el funcionamiento social; limitaciones emocionales durante el rendimiento en el trabajo, autocaracterizaciones como nervioso, y disfrute reciente de vida.

b) Escala de bienestar psicológico. A partir de Cummins (1993, citado en Veenhoven, 2006) la calidad de vida se ve como un conglomerado de componentes objetivos y subjetivos, donde cada uno se divide en los siguientes siete dominios: bienestar material, salud, productividad, intimidad, seguridad, lugar en la comunidad y bienestar emocional.

c) Una medida sociológica de calidad de vida individual. Una de las primeras tentativas de representar la calidad de vida en una población general se hizo con el Estudio de prosperidad comparativo en Escandinavia, en 1976, en el que la prosperidad se midió bajo los criterios de ingresos, vivienda, apoyo político, relaciones sociales, condición de irremplazable, hacer cosas interesantes, salud y educación. 
d) Una medida de calidad de vida en las naciones. El índice de desarrollo humano (PNUD, 2014) es el criterio principal usado en estos informes a partir de indicadores de: salud pública, medida por el poder adquisitivo por cabeza; educación, medida por la alfabetización y escolaridad; y esperanza de vida en el nacimiento.

Numerosos estudios realizados en el mundo muestran las conexiones entre la satisfacción con la vida a propósito de las variables sociodemográficas; incluso se ha llegado a establecer comparaciones entre países y tipos de culturas. Algunos ejemplos:

Las variables sociodemográficas como edad, sexo, ingreso y educación no explican más del $10 \%$ de la varianza en felicidad individual, al menos en las naciones ricas. En el caso del ingreso, en estados de bienestar ricos normalmente encuentran pequeñas correlaciones, pero en otros países se observan diferencias bastante sustanciales. Cuanto más pobre sea la nación, más altas tienden a ser las correlaciones (Veenhoven, 1994). Al respecto, North, Holahan, Moos y Cronkite (2008, citados en Martínez \& Ros, 2010) encontraron una relación muy débil de los ingresos y la felicidad, mientras que Georgellis, Tsitsianis y Ping Yin (2009, citados en Martínez \& Ros, 2010) sí hallaron una relación positiva entre el nivel de ingresos y la satisfacción con la vida.

En cuanto a la correlación con educación, los países más educados son los más felices; hay incluso más consistencia en la correlación con el trabajo. En todo el mundo, los profesionales y los directivos tienden a estar más satisfechos con la vida. Por otra parte, el estado civil produce más satisfacción con la vida en las naciones occidentales más modernas como Dinamarca o los Países Bajos, en oposición a países más tradicionales como Irlanda o Italia (Veenhoven, 1994). Según este autor, la satisfacción con la vida está también relacionada de forma bastante consistente con la presencia y calidad de los lazos íntimos; en las naciones occidentales, el matrimonio es más relevante que los contactos con amigos y parientes; las correlaciones más importantes tienen que ver con la capacidad personal para afrontar los problemas de la vida. La satisfacción con la vida tiende a ser mayor entre las personas que gozan de una buena salud física y que tienen mucha energía; los satisfechos también comparten las características de buena salud mental y resistencia psicológica.

Con respecto a la personalidad, el satisfecho tiende a ser extrovertido socialmente y abierto a la experiencia. Hay una tendencia notable hacia creencias de control interno, mientras que las personas que están insatisfechas tienden a pensar que son un juguete del destino. Muchos de los hallazgos sobre la variación individual en satisfacción con la vida se reducen a una diferencia en la capacidad de controlar el entorno propio. En las modernas sociedades occidentales individualistas, posiblemente esto es más común: la varianza normal explicada por tales varia- 
bles personales tiende a estar cerca del $30 \%$ (Veenhoven, 1994).

Los factores culturales y sociales influyen en el BS en varias formas; algunos países son más capaces de satisfacer las necesidades básicas de su población, tales como alimentos, agua limpia, y la salud; en estas naciones se evidencian mayores niveles de bienestar subjetivo. También existe una correlación entre la cultura y el BS, ya que influye en las metas y los valores de las personas. Además, las variaciones en la influencia cultural en los niveles medios de BS parece que obedecen a diferencias en el optimismo y positividad, el apoyo social, patrones de afrontamiento, y el grado de regulación del individuo (Diener, 2000). Por ejemplo, según Diener (1996, citado en Barreat, 2011), las personas que pertenecen a una cultura individualista se sienten más satisfechas que aquellas orientadas de manera colectivista. En naciones individualistas las personas tienden a referirse a sus propios afectos y sentimientos como indicadores de su calidad de vida; mientras que en culturas colectivistas, el nivel de satisfacción está dado por la evaluación que se hace de los aspectos sociales y familiares.

En ese mismo sentido, un estudio desarrollado por Veenhoven (1999, citado en Barreat, 2011) comparó la calidad de vida percibida en 43 naciones. Los resultados demostraron una relación positiva y significativa, ya que mientras más individualizada es la nación, más disfrutan los ciudadanos sus vidas; en los países colectivistas; la persona es moldeada de acuerdo con las demandas de la sociedad.
El temperamento y la personalidad parecen ser potentes factores que influyen en el BS de la gente, en parte porque por lo general los individuos se adaptan a un cierto grado de buenas y malas condiciones. También los valores y objetivos de las personas parecen estar íntimamente ligados al BS, por lo que una hipótesis plausible es que el cambio es un componente objetivo inherente de adaptación (Diener, 2000).

Por otro lado, Arita (2005) buscó integrar en un estudio a los indicadores objetivos y subjetivos de la capacidad junto con los indicadores del bienestar subjetivo (satisfacción por la vida y felicidad sentida), para considerarlos como dimensiones explicativas de la calidad de vida.

Con respecto al enfrentamiento (también conocido como afrontamiento) a los problemas, después de la aparición en los ochenta del modelo de estrés de Lazarus y Folkman (1984, citados por Vázquez, Crespo \& Ring, s.f.) se hizo patente la necesidad de evaluar el afrontamiento como un proceso en relación con la situación concreta y la valoración que el sujeto hace de ella. El enfrentamiento es definido por Lazarus y Folkman (1984b, citados en Folkman, Lazarus, DunkelSchetter, DeLongis \& Gruen, 1986) como un constante esfuerzo en el cambio cognitivo y conductual de la persona para gestionar la demanda externa o interna que excede sus recursos.

La formulación teórica de estrés y enfrentamiento muestra tres importantes aspectos: la creencia de la persona acerca de que tiene el con- 
trol en una transacción de estrés puede aumentar la amenaza; la relación entre el control y la superación; y los caminos por los cuales el control puede afectar los resultados de los encuentros estresantes (Folkman, 1984). Por su parte, Lazarus (1986, citado en Vázquez, Crespo \& Ring, s.f.) señala que el afrontamiento debe referirse a un contexto específico; observar los cambios temporales, y adoptar un enfoque longitudinal, para estudiar esos cambios.

Los estilos derivados de la propuesta teórica de Lazarus y Folkman, según Vera, Laborín, Domínguez y Peña (2003) son:

- Directo: cuando las personas expresan hacer algo, ya sea cognoscitiva o conductualmente, para resolver el problema.

- Emocional: cuando las personas expresan ante un problema tener un sentimiento, una emoción o una manifestación cognoscitiva conductual de ese sentimiento o emoción.

- Evasivo: cuando las personas expresan algo para escapar del problema o evitarlo, para no ver su importancia, ya sea de manera cognoscitiva o conductual.

- Revalorativo: cuando las personas expresan darle un sentido positivo al problema, de alguna manera mejorar su percepción.

- Directo social: cuando las personas solicitan ayuda de alguien o comparten con alguien sus ideas u opciones para resolver el problema (p.15).

Las estrategias propuestas por Lazarus y
Folkman (1984, citados en Nava, Ollua, Vega \& Soria, 2010) son las dirigidas a la acción, es decir, todas aquellas actividades o manipulaciones orientadas a modificar el problema; y aquellas dirigidas a la emoción, como son las acciones que ayudan a regular las respuestas emocionales a las que da lugar el problema.

Con el propósito de investigar las principales formas de enfrentamiento a diferentes situaciones de problemas de sujetos yucatecos, en México se siguió un muestreo no probabilístico por cuotas que contemplaba a 790 sujetos yucatecos (45,5\% hombres y 54,5\% mujeres) de la ciudad de Mérida, Yucatán, de nivel socioeconómico bajo-alto y medio-bajo. Los factores que obtuvieron mayor puntuación fueron los directosrevalorativos seguidos de los emocionales-negativos y los evasivos (Góngora \& Reyes, 1999).

A partir de un estudio sobre la población en el centro del país respecto al bienestar subjetivo, autoconcepto, locus de control, orientación al logro, evitación al éxito y enfrentamiento a los problemas (Vera, et al., 2003) se procedió a investigar su validez y las diferencias por género, edad y educación en el Estado de Sonora. Los resultados evidenciaron la sensibilidad y precisión de las escalas, así como diferencias por grupo de edad, sexo, ocupación y escolaridad, y se reafirmó el papel que juega el bienestar subjetivo en la percepción de control y en los estilos de enfrentamiento que la persona manifiesta en las distintas situaciones o escenarios en los que participa. 
En cuanto a los antecedentes en torno a los docentes universitarios, se encontraron algunos estudios que dan cuenta de aspectos relacionados con su CV y sus actividades académicas:

En la Universidad de Alicante se analizó el grado de satisfacción que refieren los profesores universitarios con aspectos específicos y centrales de sus tareas académicas. Sus resultados indican que las dos actividades académicas que producen mayor satisfacción entre el profesorado son la docencia y la relación con los estudiantes. Se encontraron diferencias estadísticamente significativas en algunas de las tareas académicas examinadas debidas al género o categoría académica de los profesores, pero no en la edad del profesorado (Herranz-Bellido, Reig-Ferrer, Cabrero-García, Ferrer-Cascales \& GonzálezGómez, 2007).

Con la utilización de la hermenéutica crítica, aplicando los principios de la complejidad, a partir de una revisión documental, Guevara y Domínguez (2011) concluyeron que la calidad de vida del docente universitario ha emergido como una realidad en deterioro progresivo respecto a los componentes socioeconómicos, de salud mental y satisfacción laboral, por lo que son relevantes el trabajo, la recreación, el apoyo social y familiar como compensadores.

En la Universidad de Colima (México) se realizó una investigación con 189 profesores universitarios (71 \%, hombres y $29 \%$, mujeres), en la que se concluyó que independientemente del sexo, salario o estatus laboral de los profesores universitarios, es la salud el factor principal que modifica la mayor cantidad de rubros de la calidad de vida (Verdugo, Guzmán, Moy, Meda \& González, 2008).

En la Universidad de Lleida, España, se desarrolló una investigación con docentes, quienes mostraron su percepción personal de malestar, con un síndrome de burnout moderado o intermedio, superior en mujeres y en profesores de menor escalafón y antigüedad. Se encontró un nivel de insatisfacción moderado y cierto grado de apatía, indicado sobre todo en el ámbito de las relaciones personales, asociándose con una mayor percepción de problemas de salud (Sánchez Fernández \& Clavería Señis, 2005).

En un estudio fenomenológico llevado a cabo con docentes universitarios jubilados de Guadalajara, Jalisco, México, acerca de su jubilación, estado de salud, calidad de vida y la participación de la familia, la mitad de los entrevistados consideraron tener una buena calidad de vida. Las mujeres mostraron una mayor capacidad de adaptación al ambiente familiar posterior a la jubilación, mientras que el hombre encuentra dificultades en su inserción al hogar ante la ruptura de su rol como proveedor económico y jefe del hogar (Rodríguez \& Aguilar, 2005).

En Venezuela, González (2008) mide la prevalencia del estrés en la satisfacción laboral de docentes vinculados a institutos de Tecnología de Cabimas y Maracaibo, usando la hermenéuti- 
ca. Los resultados indicaron que el distrés laboral (estrés malo) prevalece en una tercera parte de los entrevistados ante las demandas excesivas de trabajo. Se constató que están satisfechos con el trabajo la tercera parte de los profesores, y son los factores intrínsecos los determinantes de la satisfacción con el trabajo que afirma su individualidad.

Los problemas de conducta de los alumnos, la sobrecarga laboral y el conflicto de rol laboral están presentes en los centros escolares, y probablemente sean inevitables. Cuando las situaciones generadoras de estrés son difíciles de controlar por parte de la institución educativa, la estrategia de incrementar la autoeficacia mediante el desarrollo y puesta en práctica de estrategias de afrontamiento adecuadas frente a las demandas del trabajo puede contribuir a reducir el estrés docente y fomentar un mayor bienestar y satisfacción laboral entre los profesores (GismeroGonzález, et al., 2012).

Gran parte de los estudios empíricos exponen la complejidad del estudio de la calidad de vida y muestran que se deben considerar las dimensiones: objetiva, cuya orientación está dirigida a los recursos y que representa la capacidad de la persona para hacer frente a la vida; y subjetiva, que está más orientada a las necesidades del individuo y que, a su vez, tiene dos componentes, el cognitivo (satisfacción con la vida global o en dominios específicos) y el afectivo (afectos positivos y negativos).

\section{Propuesta de modelo de calidad de vida}

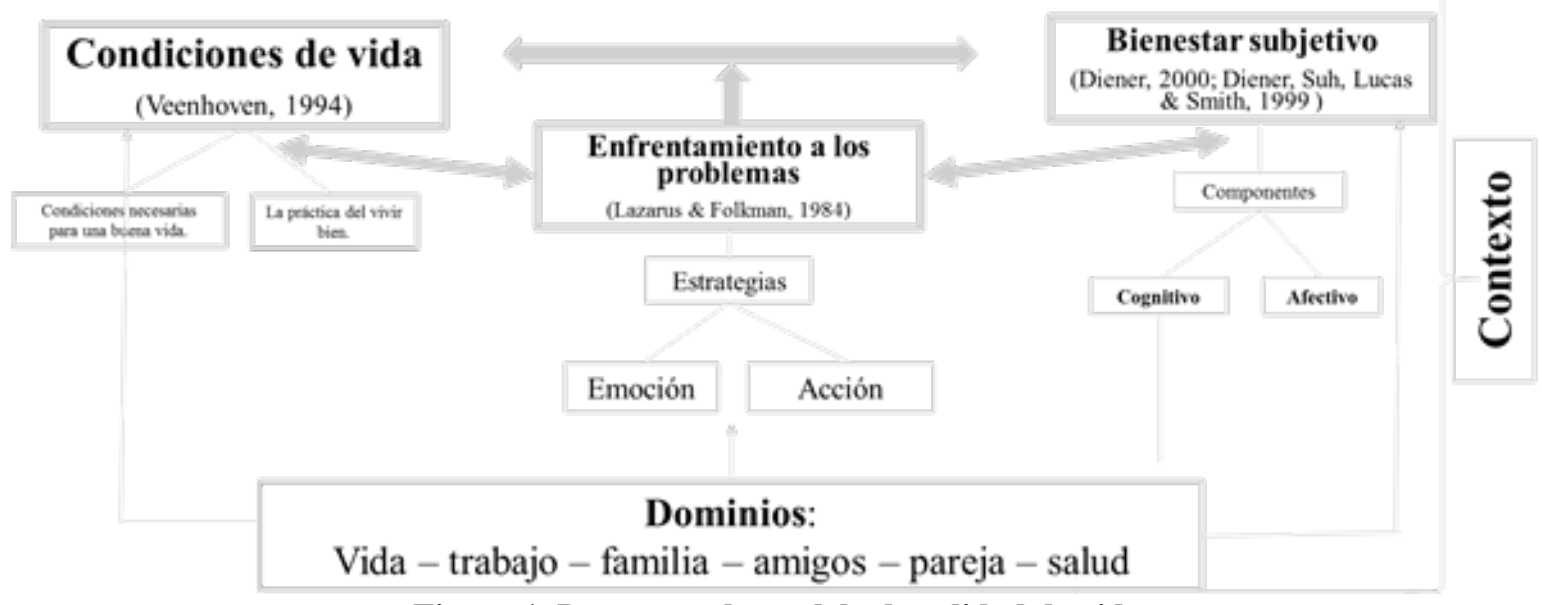

Figura 1. Propuesta de modelo de calidad de vida

Fuente: Elaboración propia, con base en Veenhoven (1994); Diener, Suh, Lucas y Smith (1999); Diener (2000); Lazarus \& Folkman (1984) 
En la satisfacción con la vida, intervienen particularmente aspectos como el ingreso, la cultura, la edad, el género, el estado civil, si brindan o reciben apoyos, pero también el tipo de estrategias con que enfrentan los problemas. Sobre esto último, poco se ha estudiado en relación a los diferentes componentes de la calidad de vida. Al respecto, Góngora (1998) afirma que existe evidencia de que las características de personalidad influyen en la forma como se enfrentan los problemas, pero tanto la personalidad y el enfrentamiento se dan en un contexto, que propicia o favorece determinado tipo de respuesta y este contexto puede ser específico como la situación particular que se enfrenta o más amplia y general, como la cultura.

Por último, luego de todo lo expuesto se propone el siguiente modelo conceptual, con el propósito de generar una estructura analítica que podría ser replicada en un contexto diferente al de la educación superior y con participantes que tuvieran características distintas a las de los docentes de este sistema educativo.

\section{Conclusiones}

Con base en el modelo conceptual propuesto, se plantea la importancia de realizar un estudio donde se integran los tres componentes como variables que pueden explicar la calidad de vida. Con ello se busca:

- Evidenciar la existencia de una relación significativa entre las condiciones de vida de las personas y los estilos con que enfrentan los problemas, lo cual no se ha encontrado hasta el momento en la bibliografía revisada.
- Visualizar una nueva perspectiva, alternativa, para medir la calidad de vida, que además de incorporar los componentes objetivo y subjetivo de la CV, lo que ya ha sido objeto de múltiples estudios de manera separada o conjunta, también considere los estilos de enfrentamiento como una variable que contribuye a explicar la $\mathrm{CV}$ de las personas.

- Proponer un instrumento para profundizar en las condiciones de vida de las personas, tomando las diferentes áreas de sus vidas, pues son parte de las escalas de bienestar subjetivo y de enfrentamiento a los problemas: trabajo, familia, amigos, pareja y salud. Con ello se busca complementar el análisis de lo que ha sido llamado calidad de vida objetiva o condiciones objetivas de vida, para lo cual generalmente se contemplan los indicadores: ingreso, educación y salud como base para realizar comparaciones entre naciones dejando de lado aspectos que pueden influir en el bienestar subjetivo o en la capacidad para enfrentar la vida, entre los que se encuentran: familia, pertenencia a grupos, si tienen pareja o hijos.

- Mostrar las relaciones existentes entre las condiciones de vida, el bienestar subjetivo y los estilos de enfrentamiento, variables que se fijan como independientes y que interactúan y se afectan entre sí, al mismo tiempo, que influyen en la calidad de vida, variable resultante o dependiente.

Por la multidimensionalidad del concepto de la $\mathrm{CV}$, se hace necesaria la integración de distin- 
tos enfoques disciplinarios (Cummins \& Cahill, 2000; Delgado \& Salcedo, 2008; Tonon, 2016). Si bien particularmente los modelos teóricos de las últimas décadas han relacionado el BS con el temperamento, el conocimiento de sí mismo, las metas, la cultura y el enfrentamiento, también es necesario clarificar las interrelaciones de muchos de estos factores (Diener et al., 1999).

Finalmente, se considera que aun cuando existen estudios, indicadores y profundas reflexiones sobre cómo se han ido reorientando las instituciones de educación superior bajo la lógica del mercado (Ibarra, 2009), poco se sabe acerca de las condiciones de vida de los principales actores de la IES: los docentes, ni sobre sus recursos psicológicos para enfrentar las situaciones estresantes derivadas de su trabajo académico y de otros ámbitos de sus vidas. Tampoco se ha llegado a relacionar lo anterior con el diseño de propuestas que vinculen a la universidad y sus miembros con los diferentes sectores de la sociedad, de tal manera que pueda darse una verdadera contribución al desarrollo de las regiones en donde se encuentran situadas las IES.

\section{Referencias}

Acosta, L. \& Aguilar, E. (2013). Trabajo académico y producción científica en la Universidad Autónoma de Zacatecas (UAZ). En M. E. Martínez, F. Piñero, S. Figueroa \& B. U. Aires (Ed.), El papel de la universidad en el desarrollo (pp.221-227). México. DOI: 10.13140/RG.2.1.4595.4325.
Arita, B. (2005). La capacidad y el bienestar subjetivo como dimensiones de estudio de la calidad de vida. Revista Colombiana de Psicología, (14), 73-79.

Banda C., A. L. \& Morales Z., M. A. (2012). Calidad de vida subjetiva en estudiantes universitarios. Enseñanza e Investigación en Psicología, 17(1), 29-43.

Barba, A. (2012). La calidad en las instituciones de educación superior en México: ¿De lo privado a lo público? En A. Barba \& O. Lobato, Las instituciones de educación superior desde la perspectiva del análisis organizacional (pp.135-150). México, D.F.: Universidad Autónoma Metropolitana.

Barreat, Y. (2011). Predictores de calidad de vida en distintas culturas: una aplicación de la teoría de individualismo-colectivismo. Anales, 11(1), 53-69.

Comas, O. \& Rivera, A. (2011). Los estímulos económicos y la evaluación del desempeño docente en las instituciones universitarias. Praxis Investigativa ReDIE. Revista Electrónica de la Red Durango de Investigadores Educativos A.C., 3(5), 15-26.

Cummins, R. \& Cahill, J. (2000). Avances en la comprensión de la calidad de vida subjetiva. Intervención Psicosocial, 9(2), 185198.

De Vries, W. (1996 enero-marzo). Políticas públicas y funcionarios modernos. (Instituto de Investigaciones sobre la Universidad y la Educación). Perfiles Educativos (71), $1-14$. 
De Vries, W. \& Ibarra, E. (2004, julio-septiembre). La gestión de la Universidad. Interrogantes y problemas en busca de respuestas. RMIE, 9(22), 575-584.

Delgado, P. \& Salcedo, T. (2008). Aspectos conceptuales sobre los indicadores de calidad de vida. Revista Electrónica La Sociología en sus escenarios (17), 1-32. Recuperado desde http://aprendeenlinea.udea. edu.co/revistas/index.php/ceo/article/ view/6803/6233

Diener, E. (2000). Subjective Well-Being. The Science of Happiness and a Proposal for a National Index. American Psychologist, 55(1), 34-43. DOI: 10.1037//0003066X.55.1.3.

Diener, E., Suh, E., Lucas, R. \& Smith, H. (1999). Subjetive Well-Being: Three Decades of Progress. Psychological Bulletin, 125(2), 276-302.

Fernández-López, J. A., Fernández-Fidalgo, M. \& Cieza, A. (2010). Los conceptos de calidad de vida, salud y bienestar analizados desde la perspectiva de la clasificación internacional del funcionamiento (CIF). Rev. Esp. Salud Pública, 84(2), 169-184.

Folkman, S. (1984). Personal control and stress and coping processes: A theoretical analysis. Journal of Personality and Social Psychology, 46(4), 839-852.

Folkman, S., Lazarus, R. S., Dunkel-Schetter, C., DeLongis, A. \& Gruen, R. J. (1986). Dynamics of a stressful encounter: Cognitive Appraisal, coping, and encounter outcomes. Journal of Personality and Social Psychology, 50(5), 992-1003.
Gil, M. (2012). La educación superior en México entre 1990 y 2010 . Una conjetura para comprender su transformación. Estudios Sociológicos, 30(89), 549-566.

Gismero-González, M., Bermejo, L., Prieto, M., Cagigal, V., García, A. \& Hernández, V. (2012). Estrategias de afrontamiento cognitivo, autoeficacia y variables laborales. Orientaciones para prevenir el estrés docente. Acción Psicológica, 9(2), 87-96. DOI: http://dx.doi.org/10.5944/ ap.9.2.4107.

Gobierno de Chile (2002). Calidad de vida en el trabajo: Percepciones de los trabajadores. Santiago: Dirección del Trabajo. Departamento de Estudios. Recuperado desde: http://dt.gob.cl/1601/articles-64333_ recurso_1.pdf

Góngora, E. A. (1998). El enfrentamiento a los problemas y el papel del control: una visión etnopsicológica en un ecosistema con tradición. Tesis doctoral no publicada, Universidad Nacional Autónoma de México.

Góngora, E. \& Reyes, I. (1999). La estructura de los estilos de enfrentamiento: rasgo y estado en un ecosistema tradicional mexicano. Revista Sonorense de Psicología, 13(2), 3-14

González, N. (2008). Prevalencia del estrés en la satisfacción laboral de los docentes universitarios. Revista Electrónica de Humanidades, Educación y Comunicación Social, 3(4), 68-89. 
González, S., Brunner, J. \& Salmi, J. (2013). Comparación internacional de remuneraciones académicas: un estudio exploratorio. Calidad en la Educación, (39), 22-42. DOI: http://dx.doi.org/10.4067/S071845652013000200002.

Guevara, H. \& Domínguez, A. (2011). Calidad de vida del docente universitario vista desde la complejidad. Revista Cubana de Salud Pública, 37(3), 314-323.

Haas, B. K. (1999). A Multidisciplinary Concept. Analysis of Quality of Life. Western Journal of Nursing Research, 21(6), 728742.

Herranz-Bellido, J., Reig-Ferrer, A., CabreroGarcía, J., Ferrer-Cascales, R. \& González-Gómez, J. (2007). La satisfacción académica de los profesores universitarios. Recuperado desde: http://www.eduonline. ua.es/jornadas2007/comunicaciones/2E5. pdf

Ibarra Colado, E. (2002). La nueva universidad en México: transformaciones recientes y perspectivas. Revista Mexicana de Investigación Educativa, 7(14), 75-105.

Ibarra Colado, E. (2003, septiembre). Capitalismo académico y globalización: la universidad reinventada. Educ. Soc., 24(84), 1059-1067.

Ibarra Colado, E. (2005). Origen de la empresarialización de la universidad: el pasado de la gestión de los negocios en el presente del manejo de la universidad. Revista de la Educación Superior, 34(2), 13-37.
Ibarra Colado, E. (2009). Exigencias de organización y de gestión de las universidades públicas mexicanas: de su pasado político a sus mercados presentes. En D. Cazés, E. Ibarra \& L. Porter, Las universidades públicas mexicanas en el año 2030: examinando presentes, imaginando futuros (pp.1-35). México: CEIICH-UNA,/ UAM-Cuajimalpa.

Ibarra Colado, E. \& Porter, L. (2007a). Costos de la evaluación en escenarios de continuidad: Lecciones mexicanas sobre las disputas con los mercaderes del templo del saber. Espacio Abierto Cuaderno Venezolano de Sociología, 16(1), 61-68.

Ibarra Colado, E. \& Porter, L. (2007b). El debate sobre la evaluación del homo academicus al homo economicus. Reencuentro, (48), 34-39.

Lazarus, R. S. \& Folkman, S. (1984). Estrés y procesos cognitivos. Barcelona: Ed. Martínez Roca.

López, A. \& Restrepo, F. (2011). Análisis de la subjetivación laboral sobre la calidad de vida laboral del personal docente de la Universidad de Antioquia. Tesis de maestría no publicada, Universidad de Manizales, Colombia.

Malacara, R., Cázarez, A., Galván, L. \& Ramírez, P. (2014). La evaluación de la educación superior y su relación con los retos de innovación. International Review of Business Research Papers, 10(3), 32-53.

Martínez, S. \& Preciado, M. (2009). Consecuencias de las políticas neoliberales sobre el 
trabajo y la salud de académicos universitarios: el burnout como fenómeno emergente. Psicología y Salud, 19(2), 197-206.

Martínez, M. \& Ros, R. (2010). De la calidad de vida laboral a los riesgos psicosociales: Evaluación de la calidad de vida laboral. Acciones e Investigaciones Sociales, 28, 5-55.

Martínez, S., Méndez, I. \& Murata, C. (2011). Becas, estímulos y sus consecuencias sobre el trabajo y la salud de docentes universitarios. Reencuentro: Estudios sobre educación, 61, 56-69.

Nava Q., C., Ollua M., P., Vega V., C. Z. \& Soria T., R. ( 2010). Inventario de estrategias de afrontamiento: una replicación. Psicología y Salud, 20(2), 213-220.

Palomar, J. (2005). Estructura del bienestar subjetivo: construcción de una escala multidimensional. En L. Garduño Estrada, B. Salinas Amescua \& M. Rojas Herrera, Calidad de vida y bienestar subjetivo en México. México: Plaza y Valdés.

Pérez-Luco, R., Alarcón, P. \& Zambrano, A. (2004). Desarrollo humano: paradoja de la estabilidad del cambio. Psychosocial Intervention, 13(1), 39-61.

Programa de las Naciones Unidas para el Desarrollo [PNUD] (2014). Informe sobre desarrollo humano 2013. El ascenso del Sur: Progreso humano en un mundo diverso. Recuperado desde: http://reliefweb.int/ report/world/informe-sobre-desarrollohumano-2013-el-ascenso-del-sur-progreso-humano-en-un-mundo
Ramos, M. E., Sieglin, V. \& Zúñiga, M. (2013). $\mathrm{La}$ instransparente transparencia... $\mathrm{La}$ asignación de sueldos y niveles académicos al profesorado en una universidad del norte de México. Perfiles Educativos, $35(141), 26-45$.

Rodríguez, M. \& Aguilar, M. (2005). Jubilación y calidad de vida en profesores universitarios: el rol de la familia. Estudios sobre Familias, 4, 49-62. Extraído el 10 de junio de 2016 desde http://sistemadif. jalisco.gob.mx/apps/ceninf/centro_de_informacion/FAMILIA/Jubilacion_y_calidad_de_vida_en_profesores_universitarios_el_rol_de_la_familia_MC_RODRIGUEZ_VEGA_DIF_JALISCO.pdf

Ruiz, C., Mas, Ó., Tejada, J. \& Navío, A. (2008). Funciones y escenarios de actuación del profesor universitario. Apuntes para la definición del perfil basado en competencias. Revista de la Educación Superior, 37(2), 115-132.

Sánchez Fernández, M. \& Clavería Señis, M. (2005). Profesorado universitario: estrés laboral. Factor de riesgo de salud. Enfermería Global, 4(1), 1-16. Recuperado desde http://revistas.um.es/index.php/eglobal/article/view/512

Terán, A. C. \& Botero, C. C. (2011). El capitalismo organizacional: una mirada a la calidad de vida laboral en la docencia universitaria. Cuadernos de Administración, 27(46), 9-21.

Tonon, G. (2016). Los estudios sobre calidad de vida y la perspectiva de la Psicología 
positiva. Psicología, Cultura y Sociedad, Psicodebate 10, 73-81. Extraído el 9 de junio de 2016 desde http://www.palermo. edu/cienciassociales/publicaciones/pdf/ psico10/10Psico_04.pdf

Urquidi, L. E. \& Rodríguez, J. R. (2010). Estrés en profesorado universitario mexicano. Actualidades Investigativas en Educación, 12(2), 1-21.

Urzúa M., A. \& Caqueo-Urízar, A. (2012). Calidad de vida: Una revisión teórica del concepto. Terapia Psicológica, 30(1), 61-71. Extraído el 25 de febrero de 2014 desde http://www.scielo.cl/scielo.php? pid =S $0718-48082012000100006$ \&script $=$ sci_arttext

Vázquez, C., Crespo, M. \& Ring, J. (s.f.). Estrategias de afrontamiento. Recuperado desde: http://pendientedemigracion.ucm. es/info/psisalud/carmelo/PUBLICACIONES_pdf/2000-Estrategias\%20de $\% 20$ Afrontamiento.pdf

Veenhoven, R. (1994). El estudio de la satisfacción con la vida. Intervención Psicosocial, 3, 87-116.
Veenhoven, R. (2006). Las cuatro calidades de vida. Organización de conceptos y medidas de la buena vida. En R. Veenhoven, Understanding Human Well-being. Nueva York: United Nations University Press.

Velarde-Jurado, E. \& Ávila-Figueroa, C. (2002). Evaluación de la calidad de vida. Salud Pública de México, 44(4), 349-361.

Vera, J. A., Laborín, J. F., Domínguez, S. E. \& Peña, M. O. (2003). Identidad psicológica y cultural de los sonorenses. Región y Sociedad, 15(28), 127-159.

Verdugo, J., Guzmán, J., Moy, N., Meda, R. M. \& González, O. P. (2008). Factores que influyen en la calidad de vida de profesores universitarios. Psicología y Salud, 18(001), 27-36. Extraído el 10 de junio de 2015 desde http://www.redalyc.org/ pdf/291/29118103.pdf

Villaseñor, G. (2003). La función social de la educación superior en México. La que es y la que queremos que sea. Xochimilco: Universidad Autónoma Metropolitana. 